\title{
Positive conditioned suppression after shifts in CS duration and US probability
}

\author{
DONALD MELTZER \\ Southern Illinois University, Carbondale, Illinois
}

\begin{abstract}
Rats were reinforced with a sucrose solution on a VI schedule for pressing a lever. Six CSs were presented during each session. CS duration was $2 \mathrm{~min}$ in two groups and $10 \mathrm{sec}$ in the other two groups. The probability of a food-pellet US was .5 for one of the two groups with each CS duration and 1.0 for the other. After 16 sessions, CS duration was shifted to $10 \mathrm{sec}$ and US probability was .5 for all subjects. The subjects for which preshift CS duration had been 10 sec showed positive conditioned suppression during postshift CSs. The subjects for which preshift CS duration was 2 min did not develop postshift positive conditioned suppression.
\end{abstract}

A number of studies have described the phenomenon known as positive conditioned suppression (e.g., Azrin \& Hake, 1969; Hake \& Powell, 1970; Karpicke, 1978; Karpicke, Christoph, Peterson, \& Hearst, 1977; Meltzer \& Brahlek, 1970; Meltzer \& Hamm, 1978; Van Dyne, 1971). In these experiments, rats made free operant responses for an appetitive reinforcer. While the subjects were responding, they were presented with short-duration conditioned stimuli (CSs) followed by appetitive unconditioned stimuli (USs). The USs were the same as the reinforcers in some studies and different in others. Response rates decreased during the CSs compared with pre-CS response rates. In other studies, longer CS durations were used and response rates increased during the CSs (Edgar, Hall, \& Pearce, 1981; Meltzer \& Brahlek, 1970; Meltzer \& Hamm, 1978). This rate increase during the CS has been termed "conditioned enhancement."

The response rates during the CS were also affected by the schedule of reinforcement for the operant response. Details of procedure differed, but when rats were the subjects, leaner reinforcement schedules, along with the lower response rates they produced, led to weaker positive conditioned suppression or stronger conditioned enhancement. In other words, a reduction in reinforcement frequency and response rate either led to a smaller rate decrease during the CS or shifted rate changes during the CS from decreases to increases (Edgar, Hall, \& Pearce, 1981; Lovibond, 1981; Meltzer, 1986).

In one set of circumstances, however, the rate changes during short CSs were quite different. Meltzer and Hamm (1974) conditioned three groups of rats to press a lever for reinforcement on a variable interval (VI) schedule. The rats were then presented with six 2-min CSs during each session. The subjects in the first group received a food-pellet US at the end of each CS. Those in the second group received a US after four of the six CSs. Those

Reprints may be obtained from Donald Meltzer, Department of Psychology, Southern Illinois University at Carbondale, Carbondale, IL 62901 . in the third group received US after two of the four CSs. The subjects in the first two groups responded at significantly higher rates during the CS than during the pre-CS baseline, and the increase was significantly larger for the first group than for the second. The CS duration was reduced to $8 \mathrm{sec}$ during the second part of the experiment. The subjects for which a US followed each CS increased their response rates slightly during the CS. The subjects in the other two groups responded at significantly higher rates during the $\mathrm{CS}$, relative to their pre-CS rates. Moreover, the increase in CS response rates was significantly greater as the number of sessions increased.

It was possible that the increased rate during the short CSs was caused by the fact that the US did not follow every CS. Therefore, Levinson and Meltzer (1977) compared the performance of one group of rats during 10sec CSs that were followed by a US with the performance of another group of rats in which the probability of a US's following a CS was .5. The subjects in the former group responded at significantly decreased rates during the CS, while those in the latter group responded at approximately the same rate during the CS and the pre-CS baseline. These results meant that the increased rates observed by Meltzer and Hamm (1974) during short CSs occurred because the short CSs followed prior conditioning with long CSs. However, the role of US probability was unclear. Either a partial schedule of US presentations throughout the experiment led to increased CS rates when short CSs followed long ones, or a partial US schedule was needed only when the short CSs were introduced. In the latter case, US probability was irrelevant during the first part of the experiment, in which long CSs were used. The present study examined these alternatives.

\section{METHOD}

Subjects

The subjects were 23 male hooded rats, between 90 and 120 days old at the beginning of the experiment. They were maintained on a 23$h$ food deprivation schedule. Water was always available in the animals' home cages. 


\section{Apparatus}

The rats were tested in standard operant conditioning chambers with interior dimensions of $22.2 \times 19.1 \times 19.1 \mathrm{~cm}$. The front wall of each chamber had a response lever $3.2 \mathrm{~cm}$ to the right of center and $6.4 \mathrm{~cm}$ above the grid floor, a liquid dipper $4.4 \mathrm{~cm}$ from the left wall and $1.0 \mathrm{~cm}$ above the floor, a food-pellet cup $3.0 \mathrm{~cm}$ from the right wall and $3.0 \mathrm{~cm}$ above the floor, and a cue light $15.0 \mathrm{~cm}$ above the liquid dipper. Each chamber was placed in a ventilated, sound-attenuating enclosure. White noise was always present in the experimental room. Electromechanical equipment in an adjacent room controlled the stimuli and reinforcements and recorded the responses.

\section{Procedure}

All the subjects initially underwent two to four sessions in which they periodically were given free $\mathbf{4 5} \mathrm{mg}$ food pellets or free presentations of a $10 \%$ sucrose solution (by weight). The lever was removed from the chamber during these sessions, and the subjects were observed in order to ensure that they responded promptly to the delivery of both food and sucrose. The response lever was then introduced, and it remained in the chamber for the rest of the experiment. The subjects were reinforced with $0.1 \mathrm{cc}$ of the sucrose solution for every lever press, until each subject made 30 responses in less than $30 \mathrm{~min}$. This required one to five sessions of varying duration for the various subjects. Each subject was then reinforced with the sucrose solution on a VI 30 -sec schedule for three 45-min sessions. During the next five 45-min sessions, each subject was reinforced with the sucrose solution on a VI 2-min schedule.

The subjects were then divided into four groups. The VI 2-min schedule of sucrose reinforcement for lever pressing was maintained for all subjects in each group throughout the experiment. The six subjects in the first group were presented with a 2-min light CS, six times during each session. A 45-mg food pellet followed three of the light CSs, but there was no food or other stimulus event following the other three CSs. The sequence of CSs followed by food and CSs not followed by food was changed for each session. Four different sequences were used during the course of the experiment. Mean time between CS onsets was $6.5 \mathrm{~min}$.

The five subjects in the second group received the same number of 2-min light CSs at the same intertrial intervals as did the subjects in the first group. However, each CS was followed by a food-pellet US.

There were six subjects in both the third and fourth groups; light was also the CS for these groups, but the CS duration was $10 \mathrm{sec}$. Three of the six CSs were followed by food for the third group, and, as with the first group, the sequences of CSs followed by food and CSs not followed by food were changed for each session. Four different sequences were used. All six CSs were followed by food for the fourth group. Mean time between CS onsets was $6.5 \mathrm{~min}$ as for the first two groups, but this meant that mean time between food USs was 8.5 min for the first two groups and less than $6.7 \mathrm{~min}$ for the last two groups.

After 16 sessions the procedure was changed. The CS duration was set at $10 \mathrm{sec}$ for all subjects, and food followed only three of the six CSs. In other words, all the subjects now received the same CS-US sequences as had the subjects in the third group during the preceding 16 sessions. This procedure continued for 8 sessions.

The responses were recorded for each subject during the six CSs and during an interval of equal duration just before the onset of the CS in each experimental session. Each response observation was converted to a rate measure of responses/minute for data analysis.

\section{RESULTS}

The data from the last eight preshift sessions were analyzed to determine the effects of CS duration and US probability on peformance. Subjects that received a 2 -min CS made a mean of 14.7 responses/min during the pre-CS interval and increased their response rate to 17.6 responses/min during the CS. Subjects that recieved a 10sec CS made 18.2 responses/min during the pre-CS interval and decreased their response rate to 16.0 re- spones/min during the CS. This interaction between CS duration and stimulus condition (i.e., pre-CS relative to CS) was significant $[F(1,19)=8.229, p<.01]$, and tests of simple main effects showed that both the increased rate during the 2-min CS and the decreased rate during the 10 -sec CS were significantly different from the rates of the same subjects during the respective pre-CS intervals. Simple main effects also showed that the pre-CS rates for the different stimulus duration groups were significantly different, but that there were no differences among their rates during the CS. The differences in pre-CS rates probably occurred because the interval between a food-pellet US and the beginning of the next CS was the same in both groups. Therefore, the time between food pellets was $1 \mathrm{~min} 50 \mathrm{sec}$ shorter for subjects that had 10-sec CSs, and their session durations were $11 \mathrm{~min}$ shorter. Both pre-CS intervals and CSs came earlier in the session for the subjects given 10-sec CSs than for those in the 2-min groups. On the other hand, rates decreased during short CSs and increased during long CSs, which would explain why there were no differences among the groups' CS rates.

US probability had no effect on response rate, nor was US probability a factor in any significant interactions. There were no other significant main effects or interactions in this analysis.

The data from the eight postshift sessions, during which the CS duration was $10 \mathrm{sec}$ for all subjects, were also analyzed. The subjects that had been given 2 -min CSs prior to the shift responded at 21.1 responses/min during the pre-CS interval and 21.4 responses/min during the CS. The subjects that had been given 10-sec CSs prior to the shift responded at 23.1 responses/min during the pre-CS interval and 15.2 responses/min during the CS. This interaction between preshift CS duration and current stimulus condition (i.e., pre-CS relative to $\mathrm{CS}$ ) was significant $[F(1,19)=13.68, p<.001]$. Tests of simple main effects showed that the difference between pre-CS and CS rates was not signficantly different for subjects that had experienced preshift CSs of 2-min duration. However, the lower rate during the $\mathrm{CS}$ for subjects that had experienced the 10-sec CS was significant. Figure 1 shows the pre$\mathrm{CS}$ and CS response rates during each postshift session for the two different preshift duration groups.

The analysis also revealed that the mean response rate increased significantly over the eight postshift sessions $[F(7,133)=4.01, p<.001]$. However, as Figure 1 shows, the rate change occurred because the pre-CS response rate increased over the eight postshift sessions while the CS response rate was relatively stable. This interaction between stimulus condition and sessions was significant $[F(7,133)=2.886, p<.01]$. Tests of simple main effects confirmed that the subjects that experienced 10-sec CSs prior to the shift responded at lower rates during the CS relative to pre-CS in all but the first postshift session. Moreover, the magnitude of the difference between CS and pre-CS rates was significantly greater during the last six postshift sessions than during the first two. 

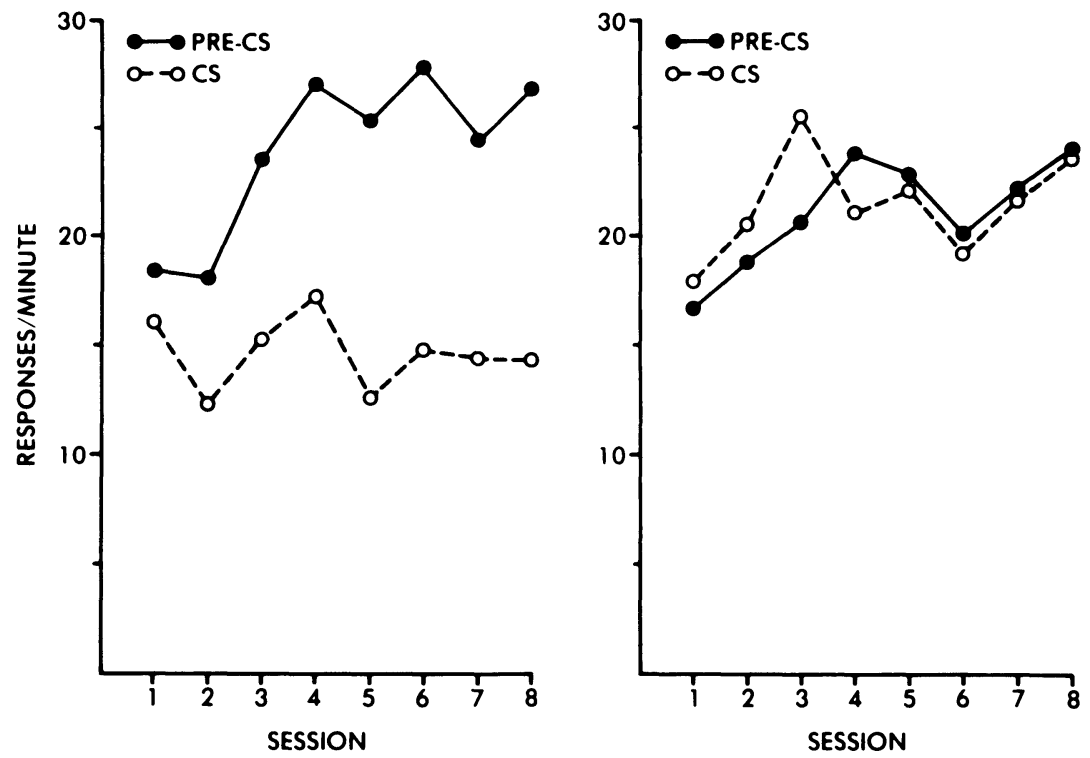

Figure 1. Mean response rates for the pre-CS interval and the CS duration in each postshift session are shown above. The panel on the left shows the mean rates of subjects for which preshift CS duration was 10 sec. The panel on the right shows the mean rates of subjects for which the preshift CS duration was 2 min.

There was no significant postshift difference between CS and pre-CS rates in any session for the subjects that experienced a 2-min CS in preshift sessions.

\section{DISCUSSION}

These results demonstrate that there were irreversible effects of prior CS duration on performance during short CSs followed by a food US on a partial schedule. The rats responded at reduced rates during short CSs, as has usually been described, but only if they had not previously experienced long CS durations. When they had experienced long CSs, they responded at approximately the same rate during the CS and baseline conditions. No such irreversible effect seems to occur when a short CS is always followed by a food US (Meltzer \& Brahlek, 1970). However, Meltzer and Hamm (1974) found that when the US probability was less than 1.0, the response rate during a short CS was much higher than the pre-CS rate in the second part of an experiment if a long CS had been used in the first part. There was no such rate increase during the short CS in the present study.

The US probability during the first part of the experiment had no effect on the difference between pre-CS and CS response rates in this study. Mean response rates were lower during the CS than during the pre-CS baseline, regardless of whether the US probability in the first part of the experiment had been .50 or 1.00 . This result may seem to conflict with the report by Meltzer and Hamm (1974), but it is easily explained by the fact that each subject in the earlier study experienced the same US probability before and after the shift in CS duration. When the CS duration was reduced, the subjects that had experienced US probabilities of less than 1.0 responded at much higher rates during the CS than during the pre-CS intervals. However, the present study showed that the same effect probably would have occurred if the US probability had been 1.0 when CS duration was long and had been reduced to less than 1.0 simultaneously with the reduction in CS duration.

There are several possible explanations for these results. The most obvious one is that contiguity between the operant response and the food US was a more common event for the subjects that were exposed to a long CS during the first part of the experiment. Consequently, superstitious conditioning would sustain high rates of the operant response when the CS duration was reduced. The objections to such a hypothe- sis are (1) that it would predict a similar result when the US probability was 1.0 in both parts of the experiment, and (2) that this does not happen (Meltzer \& Brahlek, 1970; Meltzer \& Hamm, 1974). The shift from a long to a short CS under such conditions causes the CS response rate to decrease, relative to pre-CS rates.

Another possibility is that the superstitious response is really a response suppression during the short $\mathrm{CS}$. Such a reduction could be caused by either sign tracking (Hearst \& Jenkins, 1974) or reinforcement tracking (Boakes, 1979). A reduced US probability would make such responses less likely but not impossible. If a subject experienced only short CS durations, response suppression might still develop. However, prior experience with long CS durations that caused CS response rates either to increase over baseline rates or to remain unchanged might block the development of superstitious suppression. This would explain why preshift CS duration affected the results while preshift US probability did not.

\section{REFERENCES}

Azrin, N. H., \& HAKE, D. F. (1969). Positive conditioned suppression: Conditioned suppression using positive reinforcers as the unconditioned stimuli. Journal of the Experimental Analysis of Behavior, 12, 167-173.

BoAKes, R. A. (1979). Interactions between type I and type II processes involving positive reinforcement. In A. Dickinson \& R. A. Boakes (Eds.), Mechanisms of learning and motivation: A memorial volume to Jerzy Konorski. Hillsdale, NJ: Erlbaum.

Edgar, D., Hall, G., \& Pearce, J. M. (1981). Enhancement of foodrewarded instrumental responding by an appetitive conditioned stimulus. Quarterly Journal of Experimental Psychology, 33B, 3-19.

HAKE, D. F., \& Powell, J. (1970). Positive reinforcement and suppression from the same occurrence of the unconditioned stimulus in a positive conditioned suppression procedure. Journal of the Experimental Analysis of Behavior, 14, 247-257.

Hearst, E., \& Jenkins, H. M. (1974). Sign tracking: The stimulusreinforcer relation and directed action. Austin, TX: Psychonomic Society.

KARPICKE, J. (1978). Directed approach responses and positive conditioned suppression in the rat. Animal Learning \& Behavior, 6, 216-224.

Karpicke, J., Chrisioph, G., Peterson, G., \& Hearst, E. (1977). 
Signal location and positive versus negative conditioned sppression in the rat. Journal of Experimental Psychology: Animal Behavior Processes, 3, 105-118.

Levinson, M., \&eltzer, D. (1977). Positive conditioned suppression as a function of the percentage of CS-US pairings. Bulletin of the Psychonomic Society, 10, 142-144.

LoviBond, P. F. (1981). Appetitive Pavlovian-instrumental interactions: Effects of inter-stimulus interval and baseline reinforcement condtions. Quarterly Joumal of Experimental Psychology, 33B, 257-269.

Meltzer, D. (1986). CS duration and reinforcement schedule effects on conditioned enhancement and positive conditioned suppression. Bulletin of the Psychonomic Society, 24, 290-293.

Meltzer, D., Brahlek, J. A. (1970). Conditioned suppression and conditioned enhancement with the same positive UCS: An effect of
CS duration. Journal of the Experimental Analysis of Behavior, 13 , 67-73.

Meltzer, D., \& Намм, R. J. (1974). Conditioned enhancement as a function of the percentage of CS-US pairings and CS duration. Bulletin of the Psychonomic Society, 4, 467-470.

Meltzer, D., \& Hamm, R. J. (1978). Differential conditioning of conditioned enhancement and positive conditioned suppression. Bulletin of the Psychonomic Society, 11, 29-32.

VAN DYNe, G. (1971). Conditioned suppression with a positive US in the rat. Journal of Comparative \& Physiological Psychology, 77, 131-135.

(Manuscript received April 24, 1988.) 